\title{
Cosmovisión de salud y alimentación en la cultura Guambiana
}

\author{
Worldview of health and food in the Guambiana culture \\ Nancy Janneth Molano-Tobar ${ }^{*}$ orcid.org/ 0000-0003-1953-4101 \\ Dolly Ximena Molano-Tobar ${ }^{2}$ orcid.org/0000-0002-8550-7331
}

1 Universidad del Cauca. Popayán, Colombia

2 Universidad Cooperativa de Colombia. Santiago de Cali, Colombia

Fecha de recepción: Marzo 14 - $2017 \quad$ Fecha de revisión: Octubre 31 - $2017 \quad$ Fecha de aceptación: Diciembre 21 - 2017

Molano-Tobar NJ, Molano-Tobar DX. Cosmovisión de salud y alimentación en la cultura Guambiana. Univ. Salud. 2018;20(1):16-25. DOI: http://dx.doi.org/10.22267/rus.182001.105

\begin{abstract}
Resumen
Introducción: La cultura indígena en Colombia representa un conjunto de memorias históricas, sus costumbres revelan la evolución de la cultura propia, siendo la salud y la alimentación, aspectos de importancia desde los diferentes contextos. Objetivo: Evidenciar la cosmovisión de los procesos de salud y la alimentación en la cultura indígena guambiana. Materiales y métodos: Estudio cualitativo con enfoque etnográfico con técnicas de entrevista en profundidad a 12 representantes de la comunidad establecida por el Cabildo indígena. Resultados: Del proceso investigativo sobresalieron 3 categorías: a) la cultura ancestral, nuestra herencia y orgullo b) la salud mediada por la naturaleza c) la alimentación foco de sobrevivencia y cambio. Conclusiones: Los guambianos manifiestan una riqueza cultural que se evidencia desde sus ancestros en el lenguaje y vestimenta, la cual es una forma de identidad, en el mismo sentido la salud es concebida como el equilibrio de ellos y la naturaleza, la cual les confiere no solo la posibilidad de sanación de las enfermedades desde los conocimientos milenarios de los curanderos, sino que también la madre tierra les ofrece la posibilidad de alimentarse y conservar la salud, pese a la vinculación de costumbres externas.
\end{abstract}

Palabras clave: Cultura; población indígena; salud; alimentación; estilo de vida. (Fuente: DeCS, Bireme).

\begin{abstract}
Introduction: The indigenous culture in Colombia represents a set of historical memories, where their customs reveal the evolution of their own culture, being health and food important aspects from different contexts. Objective: To demonstrate the worldview of health and food processes in the Guambiana indigenous culture. Materials and methods: A qualitative study with an ethnographic approach was conducted with in-depth interview techniques to 12 representatives of the community established by the indigenous Cabildo. Results: The following 3 categories emerged from the investigative process: a) The ancestral culture, our inheritance and pride B) the health mediated by nature C) The feeding focus of survival and change. Conclusions: The Guambiano people manifest a cultural richness that is evidenced from their ancestors in language and clothing, which is a form of identity. In the same sense, health is conceived as the balance of them and nature, which gives them not only the possibility of healing of the diseases from the millennial knowledge of the healers, but also Mother Earth offers them the possibility of feeding and preserving health, despite the linking of external customs.
\end{abstract}

Keywords: Culture; indigenous population; health; feeding; life style. (Source: DeCS, Bireme). 


\section{Introducción}

Colombia es reconocida a nivel mundial por su riqueza y diversidad de ambiente, fauna y flora, es catalogada como una "nación multiétnica y pluricultural"(1); particularmente, en el departamento del Cauca convergen 8 etnias indígenas: los yanaconas, Ingas, kokonukos, los totoroes, paeces, guambianos, eperara y siapidara, siendo la cultura guambiana una de las más representativas de la región(2), lo que plantea la necesidad de abordar los temas desde una cosmovisión de esta cultura; teniendo como base que ella "engloba los modos de vida, ceremonias, arte, invenciones, tecnología, sistemas de valores, derechos fundamentales del ser humano, tradiciones y creencias"(3). Desde esta perspectiva se busca evidenciar la cosmovisión de los procesos de salud y la alimentación en la cultura indígena guambiana, entendiendo por cosmovisión de acuerdo a Fanjul de Marsicovetere et al.(4), "la manera de ver el mundo" o en palabras de Sánchez(5) "una metanarrativa" que utilizan las personas para explicar su realidad.

La diversidad cultural que posee Colombia se representa entre otras, en las comunidades indígenas que habitan el territorio, destacando que el Departamento del Cauca, específicamente en el municipio de Silvia, se ubica el resguardo de Guambia, el cual aloja a la comunidad Misak conformada por 23.462 personas, de las cuales $77 \%$ viven en el resguardo(6), su lenguaje predominante es la lengua guambiana, wampimisameta-wam o "lengua de los hombres guambianos". El nombre guambiano (Misak) significa "hijos del arcoíris y del agua", por ello su característica particular de ubicar su resguardo cerca de los ríos y riachuelos. Su estructura está determinada por los cabildos indígenas que rigen la vida política y organizacional de las veredas articulándolas a una misma comunidad. Los líderes están representados en los Taitas quienes están comprometidos cultural, ambiental, social y políticamente con el pueblo y comparten la responsabilidad con las mamas y jóvenes líderes(7); se ha observado que la mujer hoy en día se le ha dado una mayor relevancia, pues en épocas anteriores aunque eran consideradas como la fuentes de vida(8), su participación en los procesos sociales era poco sobresaliente.

La riqueza cultural que ofrecen las comunidades indígenas permite entender a la modernidad, su actuar y hacer en relación a los procesos de la vida cotidiana y contrastarlos con la influencia de la globalización, que ha permeado a las comunidades llevando según Vergara a la aceptación de "las diferencias pero, al mismo tiempo, se procede trasformar aquellos elementos que se consideran perjudiciales dentro de las culturas o grupos sociales ${ }^{(9)}$, planteándose en varios escritos la lucha continua de las comunidades indígenas por mantener su cultura y costumbres.

El concepto de cultura abarca diferentes dimensiones entre ellas la salud, desde esta concepción la Organización Mundial de la Salud (OMS), ha planteado diferentes definiciones al término de salud, inicialmente en 1948, se definía como "ausencia de enfermedad", hoy en día se entiende "como un recurso para la vida y no como el objetivo de la vida, en el cual estar sano es la capacidad (...) para mantener un estado de equilibrio apropiado a la edad y a las necesidades sociales"(10).

Cardona(11), plantea que la salud debe observarse desde una diversidad amplia incluyendo aspectos biológicos, históricos, sociales, ambientales y espirituales que son mediados por los estilos de vida de las personas, es por ello que resulta interesante evidenciar las concepciones que presenta la comunidad indígena, teniendo en cuenta los contextos rurales en los cuales viven las comunidades, especialmente las indígenas que han asumido diferentes prácticas para la conservación y el restablecimiento de la salud, buscando a los chamanes, brujos, adivinos y hechiceros, considerándolos como personas de importancia para la comunidad(12). Castro et al.(13), indican que el Murbik el curandero guambiano "es la persona que conoce las plantas medicinales y curativas, previene enfermedades y actúa como intermediario entre los hombres y los espíritus, guiando el alma de los muertos a su nueva morada". 
Con base en lo anterior, es que las comunidades indígenas manifiestan que la pérdida del equilibrio entre la salud y la enfermedad, se debe a espíritus malos que rodean al individuo y perturban la naturaleza (madre tierra), en este sentido, la comunidad indígena asocia que los problemas de salud con la afectación que el medio ambiente ha sufrido a lo largo de la historia(14); ya que el hombre ha perdido la consciencia y el respeto hacia la naturaleza, lo que en palabras de Rosales-Rivadeneira et al.(15), la salud se ve como una conexión entre todo lo que rodea al hombre, entendida por D'Alessndro y González(16), como una ecocosmología "conjunto de conocimientos, normas y valores fundamentales para permitir la conectividad entre humanos y naturaleza".

Es indudable que la salud es regida por numerosas acciones como se observó en párrafos anteriores y haciendo una aproximación desde otra perspectiva asociada a la salud, se muestra que la alimentación forma parte trascendental en el proceso de salud, entendiendo que "las pautas de alimentación de todo grupo humano se establecen en función del medio en que se desarrolla, pues de la naturaleza, el clima y la orografía dependen la flora y la fauna que sirven como alimento a las sociedades"(17). Ante ello, las comunidades indígenas han realizado un cambio en sus hábitos alimentarios, pasando de consumir "alimentos propios de la región y preparados por ellos, a la vinculación de alimentos procesados con aditivos"(18), esto ha llevado a la Organización de las Naciones Unidas para la Agricultura y Alimentación (FAO), a identificar que "las comunidades indígenas presentan un mayor riesgo de inseguridad alimentaria y malnutrición que otros grupos, ya que padecen de niveles más elevados de pobreza, menor disponibilidad de recursos, y una creciente dependencia de alimentos más baratos, aunque con un alto grado de procesamiento"(19).

Con base en lo anterior se demuestra que "las inmigraciones constituyeron vasos comunicantes que fomentaron cambios alimentarios pero en los casos de los pueblos indígenas han traído consecuencias en la salud nutricional"(20), comprobando que "la alimentación indígena estaba íntimamente relacionada con diversas enfermedades, entre ellas la desnutrición"(12); del mismo modo, se evidencia un alto índice de enfermedades foráneas como la diabetes, la hipertensión arterial y las dislipidemias(21).

Lo planteado confirma la necesidad de evidenciar las concepciones que los indígenas guambianos tienen acerca de la salud y la alimentación, pues como menciona Villalobos et al.(22), la calidad de los alimentos que consumen los indígenas se ha deteriorado, conllevando a reemplazar su "dieta por alimentos procesados, altos en calorías y pobres en nutrientes, propios de la cultura occidental y ampliamente consumidos en todo el país", hecho que aqueja no solo los procesos de alimentación, sino también todos los aspectos de la cultura que involucran las relaciones interpersonales, sociales y políticas, confiriendo hoy en día a la población indígena del Cauca y en especial la guambiana una cultura dual(23), proveniente de sus propias raíces y de la vinculación de una cultura social influenciada por el intercambio externo que realizan con las otras comunidades, de ello la importancia de evidenciar la cosmovisión de los procesos de salud y la alimentación en la cultura indígena guambiana, con el fin de generar espacios que fomenten a futuro una estabilidad en su calidad de vida asociada a la salud desde todas sus dimensiones.

\section{Materiales y métodos}

El estudio se realizó bajo la metodología cualitativa con enfoque etnográfico(24), donde se buscó interactuar con la población, rescatando sus valores, ideologías y expectativas. Permitió obtener información empírica en el espacio en donde se desenvuelven los acontecimientos estudiados(25), las técnicas empleadas fueron entrevistas en profundidad cuyo objetivo como lo menciona Robles(26), es "adentrarse en la vida del otro, penetrar y detallar en lo trascendente, descifrar y comprender los gustos, miedos, satisfacciones, angustias, zozobras y alegrías". De la misma manera, se realizaron grupos focales representados, en los que se requirió según Hamui-Sutton y Varela-Ruiz(27) un espacio de opinión para captar el sentir, pensar y vivir de los individuos, provocando auto explicaciones para obtener datos que permitieran entender la visión 
acerca de salud y alimentación del pueblo representado en esta comunidad.

La población participante fue determinada por el cabildo indígena de acuerdo a los disponibilidad y voluntad de sus integrantes infiriendo la participación únicamente de los hombres, previo permiso, estudio del proyecto y aceptación, llegando a ser conformada bajo indicaciones del cabildo por 2 Taitas y 10 representantes, quienes firmaron consentimiento informado para mantener la integridad y anonimidad de la información, con una edad promedio de 47 años $\pm 1,45$.

El trabajo de campo se realizó durante los meses de marzo a diciembre de 2014, supervisado y siguiendo siempre las leyes del pueblo Misak, en las cuales se emplearon 4890 horas de grabación de audio con el permiso de los entrevistados y el cabildo. El análisis de la información fue sistematizado bajo el programa MAXQDA versión 12 , posteriormente se realizó la codificación de las narrativas, logrando realizar la categorización que permitió clasificar los datos recogidos de acuerdo con unidades de contenido básicas o temáticas comunes, hasta llegar a las categorías emergentes del estudio, que consistieron en: a) la cultura ancestral nuestra herencia y orgullo, b) la salud mediada por la naturaleza, c) la alimentación foco de sobrevivencia y cambio.

En la codificación de los relatos se tuvo en cuenta los grupos a los que pertenecían las personas, si eran los Taitas se identificaron con la letra $\mathrm{T}$ y si era representantes de la comunidad con la letra $\mathrm{R}$ acompañado del número respectivo; para identificar la sesión se marcó $\mathrm{S}$ con el número respectivo al que corresponde. De la misma manera, se suscribe si el relato pertenece a las entrevistas (E) o al grupo focal (G).

\section{Consideraciones éticas}

Se tuvo en cuenta lo dispuesto en la Resolución 8430 de 1993 del Ministerio de Protección Social de Colombia(28) para investigación con personas; el proyecto fue avalado por el Comité de Ética de la Universidad del Cauca, y posteriormente se presentó al cabildo indígena de Silvia como a los representantes indígenas de la comunidad, una vez explicando y comprendido, se diligenció el consentimiento informado, infiriendo lo descrito en la Ley 1581 de 2012 donde se dan las disposiciones a la protección de los datos personales.

El trabajo de campo estuvo acompañado por los representantes indígenas, considerando el estudio con riesgo mínimo de lesión en el ámbito cultural.

\section{Resultados}

El presente trabajo es el resultado de las categorías emergentes que se presentan a continuación.

\section{La cultura ancestral, nuestra herencia y} orgullo. Para los guambianos su cultura prima ante cualquier cosa y el respeto que tienen por ella se equipara con el que le deben a sus ancestros, es así como mantener la tradición de su lengua viva y su vestimenta los fortalece como comunidad.

"Es importante que los hijos de nuestros hijos sigan manteniendo nuestras creencias y nuestra lengua 'pos' es la manera de mantener vivo nuestro pueblo (ET2S3)".

"La lengua de los hombres guambianos nos da poder y diferenciación entre los mestizos, (...) es una tradición que con el tiempo debemos mantener (ER4S1)".

El vestuario de la comunidad Misak es una representación de su pueblo y costumbres, lo portan con orgullo y permite diferenciarlos de los demás, un ejemplo de ello es el "Tampal Kuari" objeto en forma de caracol tridimensional (sombrero) que cubre su cabeza y representa la historia del pueblo, otro aspecto de su vestimenta es el "anaco" que corresponde al vestido tradicional, las mujeres usan las pecheras como collares según la tradición (Figura 1).

"Quien no sabe qué es un guambiano, cuando ve a un indio con su anaco y sobrero y bufanda y a la mujeres con su pechera, llevando con orgullo sus vestidos (GR5S4)". 
“(...) nuestros padres vistieron así y nosotros también, es una tradición $y$ costumbre que seguirá con nosotros los hijo del agua (ER8S2)".

"Unos dicen que usamos sombrero (...), no es 'pa' darnos sombra ni tampoco taparnos del sol, es el significado de 'onde' vinimos (GR6S5)".

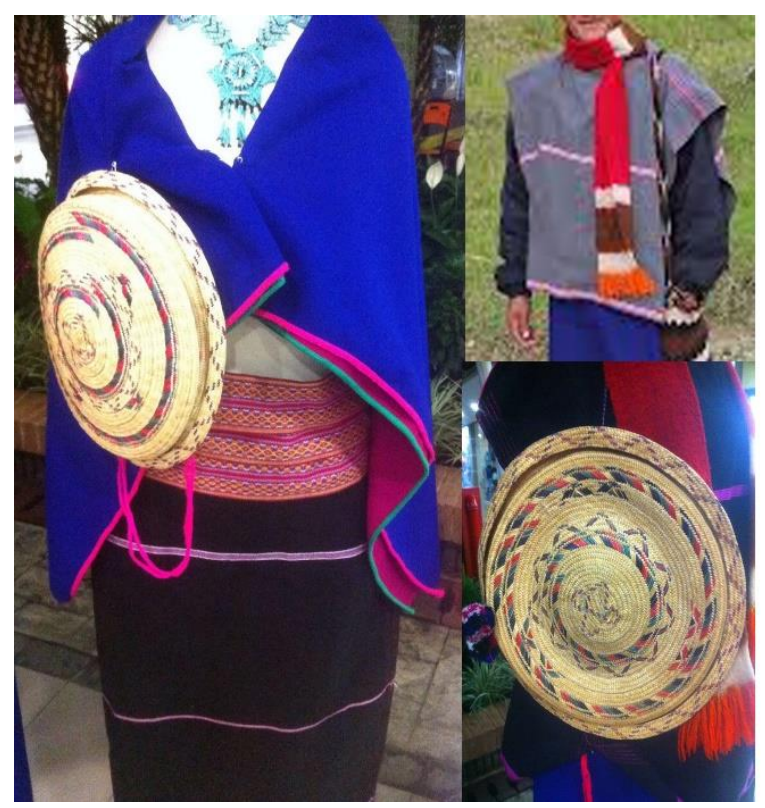

Figura 1. Vestuario guambiano

Para la cultura Misak la vivienda es importante, pues es considerada como el punto de unión de la pareja y la familia, anteriormente era la representación de la cara del ser humano desde una visión femenina, por poseer ojos y nariz construidas en bahareque que es un material compuesto de cañas o palos entretejidos y unidos con una mezcla de tierra húmeda y paja. En esta época se ha cambiado tanto la estructura como los materiales de las casas, pero su simbolismo es similar y es de vital importancia para el mantenimiento de las creencias y costumbres.

"Hace muchos años antes de conocer el ladrillo y las tejas, las casas eran de bareque y paja de forma circular y el centro era el fogón (lugar con fuego donde se cocina), 'onde' se marcaba la hora con la luz que entraba por el techo (ET1S6)".
“(..) antes se mantenía el fuego del fogón, que aparte de servirle a la mujer 'pa' cocinar, también da calor, antes era donde nos juntábamos para charlar, 'consejiar'y educar, era el punto de socialización, ahora se cambió por la estufa de gas (GT2S5)".

2. La salud mediada por la naturaleza. El concepto de salud en los pueblos indígenas especialmente el Misak, tiene una connotación integral y se relaciona con el manejo del mundo y del medio ambiente, se demuestra en estos relatos.

"La salud está ahí en las plantas, los árboles, las montañas, la 'mesma' madre tierra guarda espíritus que pueden ser benéficos o no (ET1S3)".

“(..) la salud es el equilibrio entre los somos, hablamos y decimos, pero si nos portamos mal, hablamos del otro y 'cemos' cosas en contra del otro, pos viene la mala $y$ te enfermas (ER9S6)".

“(...) provenís del agua con el equilibrio perfecto de la naturaleza, pero los espíritus malos llegan y dan enfermedad (ER3S2)".

Las creencias sobre los espíritus es fuerte entre los Misak, confiriéndoles poder sobre su salud, enfermedad y la muerte.

"Nuguwaymasig (diablo), vive en el volcán Puracé y se puede ver de diferente manera, 'tambein' hay otros espíritus Yash, Kway, Mantsik, Kalyim que sin ser malignos son temidos porque tienen la capacidad de 'quetarte' el alma de las personas, producir enfermedades y hasta la muerte (GT3S4)“.

Los curanderos llamados por los Misak, Murbik son una parte de sus tradiciones y en quien confían, limpiándolos a ellos y a sus moradas de malos espíritus, entendiendo que la madre tierra les hereda la sabiduría para mantener el equilibrio de la comunidad. 
"Los Murbik (curandero), heredan el saber de los espíritus y ellos los guían 'pa' hacer una buen trabajo, con ayuda de Pshimisak (es el principal, la unidad perfecta, encierra lo masculino y femenino), (GR5S1)".

“(...) es necesario limpiar los terrenos, las casas y uno 'mesmo', eso 'lu' hace el curandero con plantas traídas de arriba (páramo) (ER7S5)".

Los curanderos utilizan diferentes métodos para realizar la "limpias" y "Pishimar $\theta p$ " (armonización, rituales de refrescamiento), con elementos principales como plantas, que conservan su salud, que para ellos es el estado de bienestar social, físico, natural y espiritual, las clasifican como "calientes" especialmente la coca y el maíz.

"Pishimisak es el espíritu dueño de todo y la sabiduría la da él, nos da la coca que es parte del pueblo guambiano, ' $p a^{\prime}$ armonizar el equilibrio perdido (R10S7)"

“(..) aunque un refresco alcanza más o menos para seis meses puede realizarse por el advenimiento de una situación que demanda bastante trabajo de la comunidad, (...) se utiliza aguardiente (bebida de caña de azúcar fermentada), los cigarrillos, el tabaco, el agua y las plantas como coca (R10S9)".

\section{La alimentación foco de sobrevivencia y} cambio. La comunidad Misak desde sus ancestros trabajan la tierra y la consideran como fuente de su conservación, de ella provienen los alimentos que son un referente tanto cultural como social importantes, a través de la comida se genera socialización y construcción de sociedad reforzando lazos de unión y amistad, de la misma manera evidencian los cambios que la alimentación representa en su salud.

"La madre tierra, es el centro de nuestra vida, la base de nuestra organización social, el origen de nuestras tradiciones y costumbres, si la destruimos, nos destruimos a nosotros (ER8S2)".
“(...) teníamos una dieta alimentaria desarrollada en una relación armónica con los recursos del medio, los cuales hacían que el guambiano 'juera juerte ', alto $y$ gordo, ahora verás unos flacos y perezosos por comer cosas que no son de la Madre Tierra (ER1S2)".

“(..) antes se comía en abundancia maíz, ullucos (tubérculo de la región andina, proveniente de la familia Basellaceae), habas, cebolla y papas, ahora los jóvenes solo comen pasta y arroz que los enflaca y los enferma (ET2S6)".

Sus productos alimenticios giran alrededor de comida propia de la región como el maíz, ulluco y habas, entre otros.

"Uno de los platillos dentro de la gran variedad de guisos a partir del maíz, es la sopa de maíz, masitas de choclo (mezcla proveniente de la harina de maíz y agua) y chicha (bebida proveniente de la fermentación del maíz y agua en una olla de barro), todos sentados en bancos alrededor del fogón (ER2S7)".

"Recuerdo cuando 'jornaliabamos' o en las mingas (tradición de trabajo comunitario para un fin común), las mujeres nos daban tazas de café, agua de panela o mazamorra de maíz o mexicano (producto hervido de leche con maíz), que daban fuerzas suficientes 'palcanzar' a trabajar ese día (ER5S6)".

El cambio climático y la influencia en sus procesos alimenticios, ha conllevando a usar elementos que contaminan el medio ambiente, de la misma manera se ha optado por sustancias que hoy en día se brinda con los procesos industrializados.

"Desde niños comíamos leche, queso, kumis hechos en casa y envueltos en hojas de bijao (plátano), y 'hora' todo se envuelve en plástico de origen fósil, que contamina (ER7S7)". 
“(...) criábamos las gallinas de patio con maíz, 'hora' por disposiciones del gobierno los citadinos comen animales de cautiverio y con muchas hormonas malas 'pala' salud (GR9S8)".

Las enfermedades conocidas como foráneas (que en palabras de la comunidad guambiana no propias de ellos e introducidas por los blancos), han aumentado, evidenciándose hipertensión y diabetes por los cambios de alimentación en la comunidad, pero también a la falta de actividad y trabajo físico.

“(...) es común ver 'hora' la presión alta y el aumento de azúcar en la sangre, eso nunca se veía antes pues todo lo que comíamos era de la tierra, 'hora' solo se ofrece dulces y chatarra (dulces y mecato) que dañan la salud (ER9S6)".

"Solíamos desde temprano saliry ayudar en la siembra y cosecha, hacíamos más ejercicio caminado y trabajando desde pequeños, hora con la televisión y los celulares se quedan quietos los niños y jóvenes (T1S4)".

\section{Discusión}

La cultura Misak es un pueblo pujante que destaca en el Departamento del Cauca por su trabajo comunitario en pro del reconocimiento de sus ancestros y respeto de sus tierras como cultura, esto concuerda con lo descrito por autores como Castro et al.(13), quienes mencionan que la realidad de la cultura guambiana insta en la integración de su cultura y mantenimiento de sus costumbres ancestrales y por Bayona Escat ${ }^{(29)}$, donde las expresiones artísticas, ritos y creencias son el dispositivo que acciona la cosmovisión de un pueblo.

La relevancia que el pueblo Misak manifiesta por sus ancestros es visible en otros pueblos indígenas, quienes lo logran a través del reconocimiento de su cultura según lo advierte Novaro et al.(30), en la trasmisión intergeneracional sobre ser indígena, que permite la comunicación de los saberes sobre el modo de vida, artesanías y cantos religiosos, considrados como productos históricos y rasgos visibles de su identidad. Este proceso se alcanza según Zalle(31), desde la interculturalidad, la cual fortalece la creación de conocimientos transdisciplinarios, relacionando las concepciones ontológicas y epistemológicas en función de la trasmisión del conocimiento, hecho que resulta importante para la comunidad Misak.

Se evidenció que una de las representaciones propias de los guambianos y motivo de orgullo es portar su vestimenta característica, pues tiene una simbología de su herencia ancestral conexa con la madre tierra; Benciolini( ${ }^{(32)}$ interpreta que el vestido se relaciona con el cuerpo humano, donde se plasma la identidad y las relaciones consigo y los otros, al igual que para los Misak es una representación de su cercanía con la madre tierra. Adicionalmente, es también el significado de su casta o estatus, frente a esto, Lorente Fernández(33), evidencia que la población indígena ha perdido su vestimenta debido al contacto directo con el citadino y con el proceso de escolarización que está generando la desaparición de la lengua náhuatl. Para Bayona(34), el traje indígena es un emblema étnico y signo visible de identificación, pertenencia y demarcación de fronteras, que se asocia con el proceso que construye la comunidad Misak.

Otro hallazgo importante para la comunidad Misak es la representación social de su vivienda, que aunque su estructura ha cambiado, el significado de ella se mantiene, el cual corresponde al sitio de unión y resguardo de la familia, concebido como "un objeto, elaborado por el hombre para cubrir necesidades básicas de resguardo, alimentación, procreación, socialización, y desarrollo del individuo como parte de una familia y de la sociedad". Aguirre añade la condición que la comunidad tiene con la naturaleza al ver la vivienda como "la estructura material (...) que evoca las relaciones de intercambio y uso de los diferentes elementos que la conforman, estableciendo escenarios tipo, que señalan formas de acción a través de un compromiso"(35) consigo, su familia y la comunidad. Se hizo evidente que la modernidad ha generado cambios en este aspecto trayendo 
consigo la perdida de algunas costumbres frente al "fogón", los cuales según Pogodda(36), tienden a fragmentar la familia y por ende la sociedad.

Respecto a la categoría "salud mediada por la naturaleza" se pudo rescatar de las entrevistas que la salud está relacionada con el equilibrio individual y la naturaleza, y esta mediada por la interrelaciones que se generen con ellos, entre ellos y lo que los rodea; en este sentido Coria(37), afirma que para los antiguos mexicanos "la salud dependía del equilibrio entre las fuerzas corporales, naturales y sobrenaturales", las cuales influían en el estado de salud individual y comunitario; por tanto la enfermedad es para el pueblo Misak, la manifestación de los espíritus, castigo por acciones impropias, las cuales se deben a los débiles lazos que la comunidad tiene $\mathrm{y}$ al deterioro del medio ambiente(14).

La importancia que el pueblo guambiano le confiere a la salud, es un hecho asociado a sus tradiciones y conocimientos ancestrales que tienes sobre las plantas y brebajes, conocidos solo por los curanderos, quienes son entrenados y seleccionados por la misma comunidad, lo cual es confirmado por Rosales-Rivadeneira et al.(15), quienes denotan que los pueblos indígenas tienen un fuerte enraizamiento en su cultura al acudir a los curanderos, quienes usan plantas medicinales, lo que les da una fuerte conexión con sus tradiciones y permiten restablecer el equilibrio del individuo. Es por ello que el conocimiento del uso de plantas en las comunidades indígenas es muy valioso y se puede observar su relevancia en diferentes culturas, como lo presenta el estudio de Álvarez-Quiroz et al.(38), sobre el uso de plantas frías y calientes en la cultura Zoque de Tabasco (México), este conocimiento debe conservarse porque puede trascender incluso a las comunidades occidentales como lo manifiesta la investigación de Restrepo et al.(39); así, el conocimiento de estas tradiciones por parte de médicos y personal de salud, puede permitir asumir una mejor relación médico-paciente cuando se desarrollen acciones conjuntas con las comunidades indígenas(14).

Las entrevistas mostraron que los alimentos que consumen los guambianos son preferiblemente cereales y tubérculos, y por su cercanía al río la trucha, como lo reporta la investigación de Echegüe et al.(19), al manifestar que los nativos consumen con frecuencia los productos de la tierra; de la misma manera, indica que la alimentación es influenciada "por cercanía a las ciudades, que les permite mayor accesibilidad y contacto intercultural", al respecto HerschMartínez y Pisanty-Alatorre(40), mencionan que la inclusión de otras formas de alimentación "constituye una afrenta hacia las culturas alimentarias locales" denominada por Villalobos et al.(22), como "aculturación", que va acompañada de la pérdida de identidad de los pueblos originarios, propiciando un aumento de enfermedades como obesidad, hipertensión arterial y diabetes.

Romero et al.(41), demuestran que la cercanía de las comunidades indígenas a las poblaciones urbanas marcan una relación estrecha con la aparición de enfermedades crónicas como la hipertensión arterial, evidenciando que el intercambio socio-económico repercute en la salud de la comunidad indígena. Herrera-Huertas et al.(42), consideran que la población indígena es una comunidad vulnerable y plantea desde la Organización Panamericana de la Salud (OPS), la necesidad de generar evidencias acerca de la incidencia de la obesidad en las poblaciones indígenas por cuanto los parámetros para evaluar el índice de masa corporal propuestos por la OMS difieren en relación a que la talla de los indígenas es más baja que la de la población general.

Lamus y Granados(43), indican que a pesar de que no existen cifras oficiales del estado de nutrición de los indígenas en Colombia, es más el grado de desnutrición presente en las comunidades indígenas, especialmente en niños, evidenciando una necesidad clara de investigar y asegurar desde la dimensión indígena su estado de salud y la relación con el proceso nutricional que hoy en día se está planteando.

\section{Conclusiones}

La comunidad Misak mantiene una lucha constante para el mantenimiento de su cultura, viéndose reflejado en su vestimenta y lenguaje, 
que a pesar de la colonización y la modernidad los esfuerzos sobresalen.

La salud es considerada como el equilibrio entre el ser uno, la madre tierra (naturaleza) y la comunidad, pero está siendo perturbada por los cambios climáticos que se están produciendo en el planeta y por el ingreso de alimentos modificados.

Los curanderos son una expresión de la cultura guambiana y son los Murbik quienes ayudan a mantener el equilibrio entre lo terrenal y lo espiritual, apoyándose en la utilización de plantas medicinales.

Las modificaciones en la alimentación y vivienda son resultado de factores externos influenciados por la cercanía a las ciudades, adicionalmente el daño al medio ambiente, propicia la inclusión de "enfermedades foráneas" propias de los mestizos.

\section{Agradecimientos}

A nuestros compañeros de trabajo, quienes brindaron sus orientaciones y sugerencias pertinentes, nuestros estudiantes que nos acompañaron $\mathrm{y}$ apoyaron los procesos de transcripción y a cada uno de los habitantes de la comunidad de Guambia, a nuestros Taitas y amigos.

\section{Conflicto de intereses}

Ninguno declarado por los autores.

\section{Referencias}

1. Colombia. Constitución Política Colombiana. Bogotá: Colombia; 1991.

2. Usme-Romero S, Alonso M, Hernandez-Cuervo H, Yunis EJ, Yunis JJ. Genetic differences between Chibcha and Non-Chibcha speaking tribes based on mitochondrial DNA (mtDNA) haplogroups from 21 Amerindian tribes from Colombia. Genet Mol Biol. 2013;36(2):149-57.

3. Raposo M del SG. Cultura, mundo indígena y educación. Rev Teoría y Didáctica las Ciencias Soc. 2003;(8):12539.

4. Fanjul de Marsicovetere R, Gibbons J, Grazioso M del P. "No hay por qué seguir a todo el mundo" La cosmovisión de guatemaltecos no Creyentes. Rev Interam Psicol. 2014;48(2):172-82.

5. Sánchez DR. Cosmovisión postmoderna. Kairos. 2012;51:126-40.
6. Murillo Escobar DR. Seguimiento a la palabra: aproximación a los ejercicios de memoria y reflexión de las autoridades ancestrales indígenas en los Círculos de Palabra 1. Maguaré. 2016;30(2):121-47.

7. Parrado-Morales S, Isidro L. La paradoja hipócrita. Problematización de la participación política de la mujer misak. Colomb Int. 2014;80:135-70.

8. Castillo-Santana PT, Vallejo-Rodríguez ED, CotesCantillo KP, Castañeda-Orjuela CA. Salud materna indígena en mujeres Nasa y Misak del Cauca, Colombia: Tensiones, subordinación y diálogo intercultural entre dos sistemas medicos. Saude e Soc. 2017;26(1):61-74.

9. Hita SR. Salud, globalización e interculturalidad: una mirada antropológica a la situación de los pueblos indígenas de Sudamérica. Cien Saude Colet. 2014;19(10):4061-9.

10. Vergara M del C. Representaciones sociales sobre salud, de algunos grupos de jóvenes de Manizales. Rev Latinoam Ciencias Soc Niñez y Juv. 2009;7(1):105-33.

11. Cardona Arias JA. Vínculo entre mestizaje y salud en un sistema medico de una comunidad indigena Colombiana. Rev Cuba Salud Pública. 2013;39(4):65164.

12. Quintero MC. Prácticas indígenas relacionadas con la enfermedad. Aquichan. 2001;1:11-3.

13. Castro C, Cepeda C, Flórez V, Vásquez Ml. Cuidado del bebé en la cultura guambiana: una mirada desde la cosmovisión de las madres. Av en Enfermería [Internet]. 2014;32(1):114-23. Disponible en: http://search.ebscohost.com/login.aspx?direct=true\&d b=lth\&AN=111323215\&lang=pt-br\&site=ehost-live

14. Alecrim W. Servicios de Salud, Pueblos Indígenas y Prácticas Médicas. Rev Salud Pública. 2001;3(1):68-70.

15. Rosales-Rivadeneira S, Álvarez-Moreno M, Tito-Pineda P. Indigenous Cosmovision About Health and Illness in Otavalo - Ecuador. Procedia - Soc Behav Sci [Internet]. 2017;237:975-9. Disponible en: http://linkinghub.elsevier.com/retrieve/pii/S1877042 817301386

16. D’Alessndro R, González A amalia. La práctica de la milpa, el ch'ulel y el maíz como elementos articuladores de la cosmovisión sobre la naturaleza entre los tzeltales de Tenejapa en los Altos de Chiapas. Estud Cult Maya. 2017;50:271-97.

17. Elvas MS. Naturaleza, alimentación y medicina indígena en Cartagena de Indias en el siglo XVI. Memorias Rev Digit Hist y Arqueol desde el caribe. 2008;4(8):1-21.

18. Molano-Tobar NJ, Montúa FA. Las costumbres del pueblo Totoró "comunidad Tototuna". Rev Investig. 2015;25:48-58.

19. Hayes P. Estado nutricional y aspectos alimentarios de mujeres indígenas del departamento de. Rev la Univ Ind Santander. 2015;47(3):271-80.

20. Rosique J, Restrepo MT, Manjarrés LM, Gálvez A, Santa J. Estado Nutricional y hábitos alimentarios en indígenas embera de Colombia. Rev Chil Nutr. 2010;37:270-80.

21. Cardona JA, Rivera Y. Representaciones sociales sobre medicina tradicional y enfermedades foráneas en indígenas EmberaChamí de Colombia. Rev Cuba Salud Pública. 2012;38(3):471-83. 
22. Villalobos D, García D, Falque L, Fernández A, Bravo A. Acercamiento Antropológico de la Alimentación del Pueblo Indígena Añu de Sinamaica Venezuela. Antropos. 2016;35:33-43.

23. Mieles-Barrera MD, García Vesga MC. Apuntes sobre socialización infantil y construcción de identidad en ambientes multiculturales. Rev Latinoam Ciencias Soc Niñez y Juv. 2010;8(2):809-19.

24. Murcia N, Jaramillo LG. Investigación cualitativa. "La complementariedad Etnografica". Una Guia para abordar estudios sociales. Kinesis. Kinesis, editor. Armenia, Quindío: Kinesis; 2000. 228 p.

25. Murillo J, Martínez C. Investigación etnográfica [Internet]. Madrid: Universidad Autónoma de Madrid; 2010. 15 p. Disponible en: https://www.uam.es/personal_pdi/stmaria/jmurillo/I nvestigacionEE/Presentaciones/Curso_10/I_Etnografic a_Trabajo.pdf

26. Robles B. La Entrevista en profundidad: Una técnica útil dentro del campo antropofísico. Cuicuilco [Internet]. 2011;52:39-49. Disponible en: http://www.scielo.org.mx/pdf/cuicui/v18n52/v18n52 a4.pdf

27. Hamui-Sutton A, Varela-Ruiz M. La técnica de grupos focales. Investig en Educ Médica. 2013;2(5):55-60.

28. Ministerio de Salud y Protección Social. Resolución 8430. Bogotá: MinSalud; 1993.

29. Bayona Escat E. Trajes indígenas y mercancías étnicas en Los Altos de Chiapas. Cuicuilco. 2016;65:11-39.

30. Novaro G, Padawer A, Borton L. Interculturalidad y Educación en Argentina desde una Perspectiva Comparativa. Educ Real. 2017;42(3):939-58.

31. Zalle JI. Conocimiento ecológico local y conservación biológica: la ciencia postnormal como campo de interculturalidad. Íconos. 2017;59:205-24.

32. Benciolini M. Hilando al norte. Nudos, redes, vestidos, textiles. Front Norte. 2016;28(55):191-6.

33. Lorente Fernández D. Medicina indígena y males infantiles entre los nahuas de texcoco: Pérdida de la guía, caída de mollera, tiricia y mal de ojo. An Antropol [Internet]. 2015;49(2):101-48. Disponible en: http://www.sciencedirect.com/science/article/pii/S01 85122515300059

34. Bayona Escat E. Trajes indígenas y mercancías étnicas en Los Altos de Chiapas. Cuicuilco. 2016;65:11-39.

35. Aguirre MG, Fernández D. Representaciones y formas de vida. Fermentum Rev Venez Sociol y Antropol. 2011;21(21):115-33.

36. Pogodda S. As culturas de desenvolvimento e o local em Timor-Leste. Rev Crit Cienc Sociais. 2014;104(1995):151-74.

37. Coria López M. Medicina, cultura y alimentación: la construcción del alimento indígena en el imaginario médico occidental a través de la visión del doctor francisco hernández. An Antropol [Internet]. 2014;48(1):59-77. Disponible en: http://linkinghub.elsevier.com/retrieve/pii/S0185122 514704898

38. Alvarez-Quiroz V, Caso-barrera L, Galmiche-tejeda A. Plantas medicinales con propiedades frías y calientes en la cultura Zoque de Ayapa, Tabasco, México. Bol
Latinoam y del Caribe plantas Med y Aromat. 2017;16(4):428-54.

39. Restrepo LF, Gomez LM, De-Ossa GC. Conocimiento Y Consumo De Bebidas Knowledge and Aromatic Drink Consumption in Youth in the City Of Em Aromáticos Jovens Na Cidade De. Biotecnol en el Sect Agropecu y Agroindustrial. 2012;10(1):87-98.

40. Hersch-Martínez P, Pisanty-Alatorre J. Desnutrición crónica en escolares: Itinerarios de desatención nutricional y programas oficiales en comunidades indígenas de Guerrero, México. Salud Colect. 2016;12(4):551-73.

41. Romero C, Zavaleta C, Cabrera L, Gilman R, Miranda J. Hipertensión Arterial Y Obesidad En Indígenas High Blood Pressure And Obesity In Indigenous Ashaninkas. Rev Peru Med Exp Salud Pública. 2014;31(1):78-83.

42. Herrera-Huerta E V., García-Montalvo EA, MéndezBolaina E, López-López JG, Valenzuela OL. Sobrepeso Y Obesidad En Indígenas Nahuas. Rev Peru Med Exp Salud Pública. 2012;29(3):5-9.

43. Lamus F, Granados C. Estado nutricional y determinantes sociales asociados en niños Arhuacos menores de 5 años de edad. Rev Salud Pública. 2013;15(4):565-76. 